Quim. Nova, Vol. 34, No. 5, 868-873, 2011

\title{
VALIDAÇÃO COMPUTACIONAL DE MÉTODOS COMPOSTOS NO ESTUDO DE PROPRIEDADES MOLECULARES
}

\author{
Gabriel Heerdt* e Nelson H. Morgon \\ Instituto de Química, Universidade Estadual de Campinas, CP 6154, 13083-970 Campinas - SP, Brasil
}

Recebido em 16/7/10; aceito em 26/11/10; publicado na web em 9/2/11

\begin{abstract}
COMPUTATIONAL VALIDATION OF COMPOSITE METHODS IN THE STUDY OF MOLECULAR PROPERTIES. Composite methods using ONIOM and different basis sets have been used to calculate proton and electron affinities for a set of alcohols at QCISD(T)/6-311++G(2df,p) level of theory. The study was carried out considering HF, MP2 and DFT (25 exchange correlation functional) methods. The calculation performed at ONIOM2(QCISD(T)/6-311++G(2df,p):HF/6-31G(d))//ONIOM2(O3LYP/6$31 \mathrm{G}(\mathrm{d}): \mathrm{HF} / 6-31 \mathrm{G}(\mathrm{d}))$ resulted in the smallest average absolute deviation for AP and AE, 4,75 kJ/mol e 0,43 eV, respectively.
\end{abstract}

Keywords: ONIOM; proton and electron affinities; composite methods.

\section{INTRODUÇÃO}

Nas últimas décadas o aumento no desempenho computacional tem tido um enorme impacto na Química Teórica. Em adição a isso, duas importantes áreas contribuíram para este progresso: o primeiro foi o desenvolvimento e implementação de métodos de correlação eletrônica cada vez mais precisos, tal como o método Coupled Cluster, ${ }^{1,2}$ e o segundo refere-se à compreensão dos efeitos de grandes conjuntos de base e seu comportamento de convergência no que diz respeito à inclusão de funções de maior momento angular. ${ }^{3}$ Juntos, esses avanços, levaram a avaliações mais precisas de diversas propriedades moleculares, em especial relacionadas a grandezas termoquímicas. . $^{40}$

Mesmo tendo em mãos métodos sofisticados e funções de base extensas para descrever qualquer sistema eletrônico, a realização prática de cálculos que envolvam um grande número de partículas ainda é uma tarefa dispendiosa computacionalmente. Algumas estratégias têm tido sucesso e tornado possível a abordagem precisa de sistemas químicos relativamente grandes. Dentre elas destacam-se a Teoria do Funcional de Densidade, o método ONIOM e também os métodos compostos.

\section{Métodos compostos}

Os métodos compostos surgiram na década de 1980 com o objetivo de obter uma energia eletrônica para uma dada geometria molecular de equilíbrio de forma aditiva, ou seja, somando termos vindos de cada etapa do cálculo. ${ }^{4}$ Atualmente, existe uma grande variedade desses métodos sendo mais difundidos os métodos Gaussian- $\mathrm{n}^{11-17} \mathrm{e}$ os métodos do conjunto de base completo. ${ }^{18-20}$

Desde o seu surgimento, devido aos resultados confiáveis para as quantidades termoquímicas e ao tempo computacional reduzido, o número de aplicações desses métodos cresceu rapidamente. Dentre as propriedades que podem ser estudadas por esses métodos estão cálculos envolvendo: afinidades por próton, ${ }^{6,21-24}$ energias de ionização, ${ }^{21,25}$ entalpias de formação, ${ }^{26}$ energias de dissociação, ${ }^{27-29}$ energias de atomização, ${ }^{30,31}$ afinidades por elétron, ${ }^{7,32,33}$ dentre outras.
Desenvolvidos por Pople e seus colaboradores, a primeira versão das metodologias Gaussian-n (G1) ${ }^{11}$ surgiu em 1988 descrita como um novo procedimento para obtenção da energia total de moléculas na geometria de equilíbrio. Com o passar dos anos foram sendo adicionadas novas correções a essa teoria e também um conjunto maior de moléculas para a validação das mesmas. A teoria encontra-se na quarta geração sendo que cada uma possui suas variações como, por exemplo, a versão G3(MP2). ${ }^{13}$

Na metodologia G3(MP2) a geometria molecular é obtida pelo método MP2/6-31G(d) considerando todos os elétrons. Uma série de cálculos single point são realizados num nível mais alto de teoria afim de corrigir a energia da geometria de equilíbrio. A energia final para um sistema qualquer é obtida considerando-se:

$$
\begin{gathered}
E[G 3(M P 2)]=E[Q C I S D(T) / 6-31 G(d)]+ \\
\Delta E(M P 2)+\Delta E(S O)+E(H L C)+E(Z P E)
\end{gathered}
$$

O termo E[QCISD(T)/6-31G(d)] corresponde a energia resultante do cálculo single point no nível QCISD(T)/6-31G(d). A correção para a perturbação de segunda ordem $(\triangle \mathrm{E}(\mathrm{MP} 2))$ é obtida por:

$$
\Delta E(M P 2)=E(M P 2 / G T \text { Large })-E(M P 2 / 6-31 G(d))
$$

Todos os cálculos para correção da energia de equilíbrio são realizados mantendo-se os elétrons do caroço fixos. Com isso um número muito menor de configurações é calculado e, portanto, o tempo computacional é reduzido significativamente.

A correção de spin-órbita $[\Delta \mathrm{E}(\mathrm{SO})]$ é adicionada para átomos a partir do terceiro período da tabela periódica, sendo seu valor obtido experimentalmente através de técnicas espectroscópicas ou então a partir de cálculos mais precisos..$^{34,35}$

A energia de ponto zero [E(ZPE)] é corrigida pelo escalonamento $(0,8929)$ das frequências provenientes do cálculo no nível $\mathrm{HF} / 6-31 \mathrm{G}(\mathrm{d})$.

E por fim, o termo E(HLC) (higher level correction) é adicionado com o objetivo de ajustar deficiências remanescentes na energia final. Ele é calculado considerando-se as expressões: 


$$
E(H L C)=-C n_{\beta}-D\left(n_{\alpha}-n_{\beta}\right)
$$

Os termos A, B, C e D são escolhidos de tal modo que os valores finais de algumas propriedades resultem no menor erro possível em relação aos valores experimentais, e valem 9,279; 4,471; 9,345 e 2,021 mhartree, respectivamente. A Equação 3 é utilizada para moléculas e a Equação 4 para átomos e seus íons. Os termos $n_{\alpha}$ e $n_{\beta}$ representam os números de elétrons $\alpha$ e $\beta$, sendo $n_{\alpha} \geq n_{\beta}$.

A partir da energia G3(MP2), dada pela Equação 1, é possível obter os valores para entalpia e energia livre e com isso explorar uma variedade de propriedades termodinâmicas. ${ }^{13}$

\section{O método ONIOM}

A partir de meados da década de 1970, surgiram métodos conhecidos como híbridos. ${ }^{36-38}$ Esses métodos consistem na combinação de diferentes tipos de aproximações, ab initio, semiempírico e mecânica molecular, tentando assim aproveitar as vantagens de cada uma e contornando algumas de suas limitações.

A primeira implementação desta nova geração de métodos ficou conhecida como IMOMM, Integrated Molecular Orbital and Molecular Mechanics. ${ }^{39}$ Após o sucesso desse método, novos estudos foram feitos para se obter maior versatilidade nos cálculos de estrutura eletrônica. Surgiu então o método ONIOM, Our own n-layered Integrated Molecular Orbital and Molecular Mechanics,${ }^{40}$ que pode ser descrito como uma superposição de cálculos como numa "cebola". Segundo o esquema teórico desse método, qualquer sistema molecular pode ser dividido em diferentes níveis ligados seguindo uma ordem mais conveniente ao problema em questão. Cada parte pode ser tratada sobre qualquer nível de cálculo e ao integrar-se os resultados obtidos nestas partes, obtém-se uma extrapolação atingindo valores de energia mais precisos sobre todo o sistema. ${ }^{41}$

Quando dois níveis diferentes de aproximação são integrados o método ONIOM segue o modelo apresentado na Figura 1.

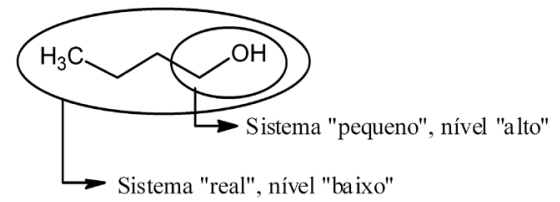

Figura 1. Diagrama esquemático do método ONIOM

A equação para a energia final de um sistema contendo 2 camadas é escrita como:

$$
E=E_{H, \text { Smodel }}+E_{L, \text { Real }}-E_{L, \text { Smodel }}
$$

em que H (high) e L (low) referem-se aos níveis de teoria "alto" e "baixo" de aproximação, respectivamente, enquanto SModel (small) e Real (real) referem-se aos sistemas de tamanho "pequeno" e "real", respectivamente. Por exemplo, pode ser realizado um cálculo no nível alto QCISD(T)/6-31G(d) para a parte pequena, e no nível baixo um cálculo semi-empírico PM3 para as partes pequeno e real, extrapolando os resultados obtém-se uma aproximação do cálculo no nível alto para a parte real, ou seja, QCISD(T)/6-31G(d) para toda a molécula.

\section{A teoria do funcional de densidade}

Em 1927 Llewellen H. Thomas e Enrico Fermi propuseram uma alternativa relativamente simples para a resolução da equação de Schrödinger, ${ }^{42}$ conhecida como a aproximação de Thomas-Fermi. Nesta aproximação, ao invés da função de onda, considera-se a den- sidade eletrônica $\rho(r)$. Assim, a energia total do sistema (E) passa a ser escrita como um funcional de $\rho(r)$, ou seja, $E[\rho(r)]$. Essa teoria passou a ganhar mais espaço em 1964-65 quando Kohn, Sham e Hohenberg formalizaram a Teoria do Funcional de Densidade. ${ }^{43,44}$

O sucesso da formalização de Kohn, Sham e Hohenberg depende da qualidade do funcional de troca-correlação de cada método. Com filosofias diferentes em suas aproximações, diversos funcionais têm sido desenvolvidos (Tabela 1). Na primeira geração de funcionais a energia de troca-correlação é obtida a partir da descrição da densidade eletrônica como um sistema de gás de elétrons homogêneo, ela é conhecida como LDA (Local Density Approximation). Os funcionais do tipo GGA (Generalized Gradient Approximation) expressam o funcional de troca-correlação em termos do gradiente de carga total. Ao adicionarem-se no gradiente de densidade eletrônica termos que dependam da densidade de energia cinética têm-se os funcionais conhecidos como meta-GGA. Uma última maneira de representar a densidade eletrônica é adicionando uma fração de energia de troca Hartree-Fock, esses métodos são conhecidos como híbridos. ${ }^{45,46}$

Tabela 1. Métodos utilizados para o estudo das propriedades moleculares

\begin{tabular}{lcc}
\hline B1LYP & PBE1PBE & MPW3PBE \\
\hline B3LYP* & tHCTH & HCTH \\
B3P86 & tHCTHhyb & HCTH147* \\
B3PW91 & X3LYP & HCTH93 \\
B972 & BMK & M06 \\
B98 & B1B95 & M06-2X \\
BhandH & MPW1LYP & VSXC \\
BhandHLYP & MPW1PBE & HF \\
O3LYP & mPW1PW91 & MP2 \\
\hline
\end{tabular}

*Métodos mantidos fixos enquanto outra variável era testada.

\section{Propriedades termodinâmicas}

O estudo de processos em que ocorre a transferência de elétrons e prótons é muito difundido devido à importância dessas reações nas mais diversas áreas, como em processos biológicos, ${ }^{47-50}$ nanoestruturas, ${ }^{51,52}$ entre outros.

\section{Afinidades por próton e eletrônica}

A afinidade por próton (AP) é definida como sendo a variação de entalpia ocorrida numa reação de desprotonação de um sistema neutro $\left(A P=-\Delta H_{6}\right)$ :

$$
H X \rightarrow X^{-}+H^{+}
$$

A afinidade por elétron (AE) é definida como sendo a variação de entalpia ocorrida numa reação em que um ânion $\left(\mathrm{X}^{-}\right)$perde um elétron ficando neutro $(\mathrm{X})\left(A E=\Delta H_{7}\right)$ :

$$
X^{-} \rightarrow X+e^{-}
$$

\section{METODOLOGIA COMPUTACIONAL}

$\mathrm{Na}$ validação de um método composto capaz de descrever as afinidades por próton e eletrônica foi utilizado um grupo de 10 monoalcoóis saturados, de cadeia alifática, linear ou ramificada e com números de carbonos variando entre 2 até 9 . Os alcoóis: etanol; 1,1-dimetil-etanol; 1-pentanol; 3-pentanol; 3-metil-2-butanol; 2-etil1-butanol; 2-hexanol; 2-metil-2-pentanol; 2-heptanol; 1-nonanol; 
foram escolhidos afim de validar um método para ser aplicado em cálculos de propriedades moleculares do colesterol, um álcool policíclico.

Os modelos testados foram: variações para o método ONIOM: ONIOM 1, ONIOM 2, sem ONIOM; funções de base dupla- $\zeta$ (duplazeta-valência) para as camadas alta (dupla- $\zeta$-a) e baixa (dupla- $\zeta$-b) do ONIOM; funções de base tripla- $\zeta$ (tripla-zeta-valência) para a camada alta do ONIOM; métodos DFT, MP2 e HF para a camada alta.

A camada alta do método ONIOM refere-se ao melhor nível de teoria aplicado, enquanto, na camada baixa o nível de teoria aplicado foi o método Hartree-Fock (HF) em todo procedimento. A escolha do método HF deve-se ao fato dele ser um método ab initio pouco custoso computacionalmente.

As etapas de cálculos realizadas para obtenção da energia para o estado fundamental foram:

- otimização e frequiência - método/dupla- $\zeta$-a:HF/dupla- $\zeta-b ;$

- energia - QCISD(T)/dupla- $\zeta$-a:HF/dupla- $\zeta-b$;

- energia: método/tripla- $\zeta: H F / d u p l a-\zeta-b$.

A energia para o estado fundamental a partir desses cálculos pode ser escrita como:

$$
E_{0}=E_{2}+E_{3}-E_{1}+Z P E
$$

sendo $\mathrm{E}_{2}, \mathrm{E}_{3}$ e $\mathrm{E}_{1}$ as energias finais obtidas nas etapas de cálculos apresentadas. Ao subtrair-se a energia obtida na etapa 1 (E1) das energias das etapas 2 e 3 (E2 e E3) obtém-se uma extrapolação para a energia no nível QCISD(T)/ tripla- $\zeta$. Foram omitidas as correções de escalonamento de ZPE e HLC, pois o objetivo foi verificar a qualidade do que está sendo testado sem "mascarar" os resultados.

As variáveis, variações ONIOM, conjuntos de funções de base e métodos, foram testadas isoladamente, ou seja, enquanto uma era testada as outras eram mantidas fixas, dessa forma foi excluída a correlação entre elas, tornando-se o procedimento mais objetivo. $\mathrm{Na}$ Tabela 1 são apresentados os métodos testados, as funções de base escolhidas são apresentadas a seguir:

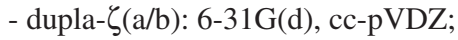

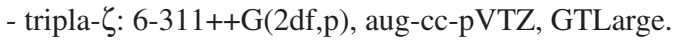

As variações escolhidas para o método ONIOM foram:

- sem ONIOM - sempre se utilizou o método e a base da camada alta para toda a estrutura;

- ONIOM 1 - as estruturas foram dividas em duas camadas, sendo que a hidroxila e o carbono diretamente ligado a ela e os átomos de hidrogênio eventualmente ligados a esses átomos foram definidos como pertencendo à camada alta, o restante da molécula foi definido como camada baixa;

- ONIOM 2 - também foram definidas duas camadas, a hidroxila, o carbono diretamente ligado a ela e os carbonos ligados a ele bem como os hidrogênios utilizados para completar valência desses átomos foram definidos como sendo da camada alta e o restante da molécula da camada baixa.
Todos os cálculos foram feitos utilizando-se os programas Gaussian03 ${ }^{53}$ e NWCHEM-5.1, ${ }^{54}$ sendo o último utilizado apenas para cálculos envolvendo funcionais de densidade não presentes no programa Gaussian03.

\section{RESULTADOS E DISCUSSÃO}

Em todos os resultados foram aplicados métodos estatísticos a fim de analisar diferenças significativas ou não entre os dados obtidos, podendo assim confirmar qual fornece os melhores valores. O método lsd (least square difference) foi utilizado para comparar médias, e ele é definido como:

$$
l s d=t_{\alpha} \sqrt{\frac{2 * M S_{\text {erro }}}{n}}
$$

onde $\mathrm{t}_{\alpha}$ é o valor tabelado de $\mathrm{t}$ de student para $95 \%$ de confiança, $M S_{\text {erro }}$ é o quadrado médio dos resíduos da ANOVA e $n$ o número de repetições em cada tratamento.

Além disso, o tempo computacional foi levado em conta, já que o objetivo também foi o de se estabelecer uma relação ótima entre custo computacional e qualidade dos resultados.

\section{Funções de base tripla-zeta-valência(tripla- $\zeta)$}

Quando testados os conjuntos de funções de base tripla- $\zeta$ obtiveramse os resultados apresentados na Tabela 2 para afinidades por próton e elétron.

Aplicando-se o teste de comparação de médias lsd para AP tem-se:

$$
\begin{aligned}
& M S_{\text {total }}=603,92 ; M S_{\text {trat }}=0,069 \\
& a(n-1)=3(10-1)=27 \\
& M S_{\text {erro }}=\frac{M S_{\text {total }}-M S_{\text {trat }}}{a(n-1)}=22,37 \\
& l s d=t_{0,025 ; 27} \sqrt{\frac{2 * M S_{\text {erro }}}{n}}=2,052 * \sqrt{\frac{2 * 22,37}{10}}=4,34
\end{aligned}
$$

onde $M S_{\text {total }}$ é a somatória de todas as variâncias, $M S_{\text {trat }}$ a somatória das variâncias dos tratamentos, $a$ o número de tratamentos e $n$ o número de repetições em cada tratamento, no caso são três funções de base $(a=3)$ e dez moléculas $(\mathrm{n}=10)$. Somando o valor obtido de $4,34 \mathrm{~kJ} / \mathrm{mol}$ com o menor desvio absoluto entre os tratamentos, tem-se um intervalo $(4,76-$ $9,10 \mathrm{~kJ} / \mathrm{mol}$ ) no qual os desvios são considerados equivalentes a $95 \%$ de confiança.

Para AE o valor obtido para o teste de lsd foi de $0,07 \mathrm{eV}$. Tanto para os desvios absolutos de AP quanto AE pode-se concluir que não existem diferenças entre as funções de base tripla- $\zeta$, isso porque nenhum desvio

\begin{tabular}{|c|c|c|c|c|c|c|}
\hline \multirow[t]{2}{*}{ Método } & \multicolumn{2}{|c|}{$6-311++G(2 d f, p)$} & \multicolumn{2}{|c|}{ aug-cc-pvTZ } & \multicolumn{2}{|c|}{ GTLarge } \\
\hline & $\mathrm{AP}$ & $\mathrm{AE}$ & $\mathrm{AP}$ & $\mathrm{AE}$ & $\mathrm{AP}$ & $\mathrm{AE}$ \\
\hline B3LYP & $5,11 \pm 4$ & $0,41 \pm 0,06$ & $4,74 \pm 4$ & $0,40 \pm 0,06$ & $5,31 \pm 3$ & $0,42 \pm 0,06$ \\
\hline НСТН147 & $5,15 \pm 4$ & $0,41 \pm 0,06$ & $4,79 \pm 3$ & $0,34 \pm 0,04$ & $4,64 \pm 3$ & $0,42 \pm 0,06$ \\
\hline Média & $5,13 \pm 4$ & $0,41 \pm 0,06$ & $4,76 \pm 3$ & $0,40 \pm 0,05$ & $4,98 \pm 3$ & $0,42 \pm 0,06$ \\
\hline Tempo relativo* & \multicolumn{2}{|c|}{0,39} & \multicolumn{2}{|c|}{1,00} & \multicolumn{2}{|c|}{0,70} \\
\hline
\end{tabular}
se encontra fora dos intervalos de confiança obtidos. Assim, o tempo computacional entra como critério para escolha da melhor função de base. Observa-se uma grande vantagem da função 6-311++G(2df,p) com relação as demais funções tripla- $\zeta$.

Tabela 2. Desvios absolutos de AP $(\mathrm{kJ} / \mathrm{mol})$ e AE (eV) para as funções de base tripla- $\zeta$

*O tempo relativo é obtido considerando-se o maior tempo como sendo $100 \%$ e o restante uma porcentagem relacionada a ele, os tempos referentes às duas propriedades e aos funcionais utilizados são considerados juntos. 


\section{Funções de base dupla-zeta-valência(dupla- $\zeta)$}

As diferentes funções de base dupla- $\zeta$ testadas nesse trabalho foram utilizadas nas camadas alta e baixo do método ONIOM, portanto, foram definidas as melhores com relação aos desvios médios absolutos e tempos computacionais para cada camada. Na Tabela 3 têm-se os resultados para as funções de base dupla- $\zeta$ para a camada alta.

Tabela 3. Desvios médios absolutos para AP $(\mathrm{kJ} / \mathrm{mol})$ e AE $(\mathrm{eV})$ para as funções de base dupla- $\zeta$ da camada alta

\begin{tabular}{lcrcr}
\hline Método & \multicolumn{2}{c}{$6-31 \mathrm{G}^{*}$} & \multicolumn{2}{c}{ cc-pvDZ } \\
& $\mathrm{AP}$ & \multicolumn{1}{c}{ AE } & \multicolumn{1}{c}{ AP } & \multicolumn{1}{c}{ AE } \\
\hline B3LYP & $5,11 \pm 4$ & $0,41 \pm 0,06$ & $11,74 \pm 5$ & $0,32 \pm 0,06$ \\
HCTH147 & $5,15 \pm 4$ & $0,41 \pm 0,06$ & $14,23 \pm 5$ & $0,35 \pm 0,07$ \\
Média & $5,13 \pm 4$ & $0,41 \pm 0,06$ & $12,98 \pm 5$ & $0,34 \pm 0,07$ \\
Tempo relativo & \multicolumn{2}{c}{0,47} & \multicolumn{2}{c}{1,00} \\
\hline
\end{tabular}

Os valores de lsd obtidos para $\mathrm{AP}$ e $\mathrm{AE}$, respectivamente, são iguais a $5,58 \mathrm{~kJ} / \mathrm{mol}$ e $0,08 \mathrm{eV}$. Sendo assim, conclui-se que a base 6-31G(d) possui resultados significativamente melhores para AP, além de um tempo computacional menor.

Na Tabela 4 são apresentados os resultados para as funções de base dupla- $\zeta$ para a camada baixa. Os valores de lsd obtidos, $5,40 \mathrm{~kJ} / \mathrm{mol}$ para AP e $0,08 \mathrm{eV}$ para $\mathrm{AE}$, deixam claro que os desvios médios absolutos não podem ser considerados diferentes. Analisando o tempo de processamento observa-se que a função de base $6-31 \mathrm{G}(\mathrm{d})$ leva uma expressiva vantagem e por isso mostra ser a mais adequada.

Tabela 4. Desvios médios absolutos para AP $(\mathrm{kJ} / \mathrm{mol})$ e $\mathrm{AE}(\mathrm{eV})$ para as funções de bases base dupla- $\zeta$ da camada baixa

\begin{tabular}{lcrrr}
\hline Método & \multicolumn{2}{c}{$6-31 \mathrm{G}^{*}$} & \multicolumn{2}{c}{ cc-pvDZ } \\
& AP & AE & AP & \multicolumn{1}{c}{ AE } \\
\hline B3LYP & $5,11 \pm 4$ & $0,41 \pm 0,06$ & $6,79 \pm 4$ & $0,39 \pm 0,06$ \\
HCTH147 & $5,15 \pm 4$ & $0,41 \pm 0,06$ & $7,23 \pm 4$ & $0,39 \pm 0,06$ \\
Média & $5,13 \pm 4$ & $0,41 \pm 0,06$ & $7,01 \pm 4$ & $0,39 \pm 0,06$ \\
Tempo relativo & \multicolumn{2}{c}{0,26} & \multicolumn{2}{c}{1,00} \\
\hline
\end{tabular}

\section{Métodos para a camada alta}

Na Tabela 5 são apresentados os desvios médios absolutos para
Tabela 5. Resultados de AP ( $\mathrm{kJ} / \mathrm{mol})$ para os métodos testados na camada alta em ordem de qualidade

\begin{tabular}{lccccc}
\hline Método & Desvios & Método & Desvios & Método & Desvios \\
\hline O3LYP & $4,75 \pm 4$ & M06 & $6,18 \pm 3$ & B3PW91 & $7,81 \pm 4$ \\
B1LYP & $4,85 \pm 4$ & mPW3PBE & $6,42 \pm 3$ & HCTH & $7,96 \pm 4$ \\
B3LYP & $5,11 \pm 4$ & B98 & $6,50 \pm 3$ & BHandH & $8,28 \pm 3$ \\
HCTH147 & $5,15 \pm 4$ & mPW1LYP & $7,16 \pm 4$ & B972 & $8,63 \pm 4$ \\
HCTH93 & $5,40 \pm 3$ & PBE1PBE & $7,34 \pm 3$ & B3P86 & $9,32 \pm 3$ \\
tHCTH & $5,64 \pm 3$ & B1B95 & $7,42 \pm 4$ & BHandHLYP & $10,19 \pm 4$ \\
VSXC & $5,64 \pm 4$ & mPW1PW91 & $7,50 \pm 4$ & MP2 & $10,28 \pm 4$ \\
X3LYP & $5,74 \pm 4$ & tHCTHhyb & $7,63 \pm 3$ & BMK & $13,31 \pm 4$ \\
M06-2X & $5,96 \pm 4$ & mPW1PBE & $7,73 \pm 4$ & HF & $23,31 \pm 4$ \\
\hline
\end{tabular}

AP em cada método testado. O valor de lsd calculado a partir desses dados é igual a 4,43 kJ/mol. Já o menor erro encontrado, para o método O3LYP, é de 4,75 kJ/mol. Assim, conclui-se que as médias aceitas são aquelas cujos valores estão entre 4,75-9,18 kJ/mol.

Os resultados obtidos para a AE são mostrados na Tabela 6. Para a comparação dos desvios absolutos pelo método lsd foram utilizados apenas os métodos aceitos na etapa anterior, ou seja, os resultados de AP. O valor obtido para esses métodos foi de $0,07 \mathrm{eV}$. Desta forma, são aceitos como sendo equivalentes desvios médios absolutos com valor máximo de $0,44 \mathrm{eV}$

Como pode ser observado dos 27 métodos testados apenas 8 (Tabela 6) foram aceitos em ambos os testes de comparação aplicados.

\section{ONIOM}

Os resultados obtidos para $\mathrm{AP}$ e $\mathrm{AE}$, considerando-se como teste os níveis da variável ONIOM, são apresentados na Tabela 7. Com os valores de 1sd, 4,29 kJ/mol para $\mathrm{AP} \mathrm{e} 0,08 \mathrm{eV}$ para $\mathrm{AE}$, pode-se concluir que não há diferença entre as variações para o método ONIOM. O tempo computacional significativamente mais baixo quando utilizadas as variações ONIOM1 e ONIOM2 indicam a versatilidade da utilização do método ONIOM nesse estudo.

\section{CONCLUSÃO}

Os resultados das avaliações das diferentes funções de base deixam claro que as funções de base de Pople, 6-31G(d) e 6-311++G(2df,p) mostram-se mais adequadas, principalmente pelo tempo computacional. Pode-se concluir que o método ONIOM1 consegue descrever tão satisfatoriamente as propriedades aqui estu-

Tabela 6. Resultados de $\mathrm{AE}(\mathrm{eV})$ para os métodos testados na camada alta em ordem de qualidade de AP

\begin{tabular}{|c|c|c|c|c|c|}
\hline Método & Desvios & Método & Desvios & Método & Desvios \\
\hline O3LYP* & $0,43 \pm 0,08$ & M06 & $0,48 \pm 0,05$ & B3PW91 & $0,51 \pm 0,06$ \\
\hline B1LYP* & $0,42 \pm 0,06$ & mPW3PBE & $0,48 \pm 0,06$ & НCTH* & $0,37 \pm 0,06$ \\
\hline B3LYP* & $0,41 \pm 0,06$ & B98 & $0,48 \pm 0,06$ & BhandH & $0,53 \pm 0,06$ \\
\hline НCTH147* & $0,41 \pm 0,06$ & mPW1LYP* & $0,38 \pm 0,06$ & B972 & $0,53 \pm 0,06$ \\
\hline НСТН93 & $0,45 \pm 0,06$ & PBE1PBE & $0,51 \pm 0,06$ & B3P86** & $0,53 \pm 0,06$ \\
\hline $\mathrm{tHCTH}$ & $0,47 \pm 0,06$ & B1B95 & $0,52 \pm 0,06$ & BHandHLYP*** & $0,55 \pm 0,06$ \\
\hline VSXC* & $0,39 \pm 0,08$ & mPW1PW91 & $0,51 \pm 0,06$ & $\mathrm{MP} 2 * *$ & $0,28 \pm 0,07$ \\
\hline X3LYP* & $0,40 \pm 0,06$ & tHCTHhyb & $0,50 \pm 0,06$ & $\mathrm{BMK}^{* *}$ & $0,54 \pm 0,09$ \\
\hline M06-2X & $0,49 \pm 0,06$ & mPW1PBE & $0,51 \pm 0,06$ & $\mathrm{HF}^{* *}$ & $0,70 \pm 0,06$ \\
\hline
\end{tabular}

*Métodos aceitos nas comparações dos desvios absolutos de AP e AE. **Métodos rejeitados na comparação dos desvios absolutos para AP. 
Tabela 7. Desvios absolutos de AP (kJ/mol) e AE (eV) para o método ONIOM

\begin{tabular}{|c|c|c|c|c|c|c|}
\hline \multirow[t]{2}{*}{ Método } & \multicolumn{2}{|c|}{ ONIOM1 } & \multicolumn{2}{|c|}{ ONIOM2 } & \multicolumn{2}{|c|}{ Sem ONIOM } \\
\hline & $\mathrm{AP}$ & $\mathrm{AE}$ & $\mathrm{AP}$ & $\mathrm{AE}$ & $\mathrm{AP}$ & $\mathrm{AE}$ \\
\hline B3LYP & $5,69 \pm 3$ & $0,45 \pm 0,06$ & $5,11 \pm 4$ & $0,41 \pm 0,06$ & $6,66 \pm 3$ & $0,49 \pm 0,07$ \\
\hline НСТН147 & $7,15 \pm 3$ & $0,49 \pm 0,06$ & $5,15 \pm 4$ & $0,41 \pm 0,06$ & $6,76 \pm 3$ & $0,47 \pm 0,07$ \\
\hline Média & $6,42 \pm 3$ & $0,47 \pm 0,06$ & $5,13 \pm 4$ & $0,41 \pm 0,06$ & $6,71 \pm 3$ & $0,48 \pm 0,07$ \\
\hline Tempo relativo & \multicolumn{2}{|c|}{0,32} & \multicolumn{2}{|c|}{0,57} & \multicolumn{2}{|c|}{1,00} \\
\hline
\end{tabular}

dadas quanto os métodos ONIOM2 ou sem utilizar ONIOM, com uma redução de tempo computacional muito significativa.

Com relação aos tipos de funcionais de troca-correlação testados foi possível observar vantagens de alguns funcionais em relação a outros, mas principalmente uma enorme vantagem do método DFT comparando aos métodos HF e MP2 (no caso da AP).

Conclui-se que a conformação ONIOM2, os conjuntos de funções de base de Pople, 6-311++G(2df,p) e 6-31G(d), e o método O3LYP resultam em desvios médios absolutos menores para $\mathrm{AP}$ e AE, 4,75 $\mathrm{kJ} / \mathrm{mol}$ e $0,43 \mathrm{eV}$, respectivamente, e vantagem em tempo computacional sendo mais adequados para descrever os sistemas em questão.

\section{AGRADECIMENTOS}

G. Heerdt agradece à CAPES pela bolsa de estudo. Ao CNPq e FAPESP pelo apoio financeiro e ao Instituto de Química da UNICAMP pela infraestrutura.

\section{REFERÊNCIAS}

1. Raghavachari, K.; Trucks, G. W.; Pople, J. A.; Head-Gordon, M.; Chem. Phys. Lett. 1989, 157, 479.

2. Pople, J. A.; Head-Gordon, M.; Raghavachari, K.; J. Chem. Phys. 1987, 87, 5968.

3. Dunning, T. H. Jr.; Peterson, K. A.; Woon, D. E.; P. v. R Schleyer, eds.; Encyclopedia of computational chemistry, Wiley: New York, 1998.

4. Lima, J. C. B.; Morgon, N. H.; Quim. Nova 2010, 33, 195.

5. Morgon, N. H.; Souza, A. R.; Sambrano, J. R.; J. Mol. Struct. 2006, 759, 189.

6. Morgon, N. H.; Int. J. Quantum Chem. 2006, 106, 2658.

7. Morgon, N. H.; Riveros, J. M.; Int. J. Mass Spectrom. 2001, $210,173$.

8. Feller, D.; Peterson, K. A.; J. Chem. Phys. 1998, 108, 154.

9. Feller, D.; Dixon, D. A.; J. Chem. Phys. 2001, 115, 3484.

10. Ruden, T. A.; Helgaker, T.; Jorgensen, P.; Olsen, J.; Chem. Phys. Lett. 2003, 371, 62 .

11. Curtiss, L. A.; Raghavachari, K.; Fox, D. J.; Head-Gordon, M.; Pople, J. A.; J. Chem. Phys. 1989, 90, 5622.

12. Curtiss, L. A.; Raghavachari, K.; Redfern, P. C.; Rassolov, V.; Pople, J. A.; J. Chem. Phys. 1998, 109, 7764.

13. Curtiss, L. A.; Redfern, P. C.; Raghavachari, K.; Rassolov, V.; Pople, J. A.; J. Chem. Phys. 1999, 110, 4703.

14. Curtiss, L. A.; Redfern, P. C.; Raghavachari, K.; Pople, J. A.; Chem. Phys. Lett. 1999, 313, 600.

15. Curtiss, L. A.; Redfern, P. C.; Rassolov, V.; Kedziora, G.; Pople, J. A.; J. Chem. Phys. 2001, 114, 9287.

16. Curtiss, L. A.; Redfern, P. C.; Raghavachari, K.; J Chem Phys. 2007, 126, 084108.

17. Curtiss, L. A.; Redfern, P. C.; Raghavachari, K.; J Chem Phys. 2007, 127, 124105.

18. Montgomery, J. A. Jr.; Frisch, M. J.; Ochterski, J. W.; Petersson, G. A.; J. Chem Phys. 1999, 110, 2822.

19. Montgomery, J. A. Jr.; Frisch, M. J.; Ochterski, J. W.; Petersson, G. A.; J. Chem Phys. 2000, 112, 6532.
20. Ochterski, J. W.; Petersson, G. A.; Montgomery Jr., J. A.; J. Chem. Phys. 1996, 104, 2598.

21. Namazian, M.; Coote, M. L.; J. Chem. Thermodyn. 2008, 40, 1116.

22. Gronert, S.; Simpson, D. C.; Conner, K. M.; J. Am. Soc. Mass Spectrom. 2009, 20, 2116.

23. Range, K.; Riccardi, D.; Cui, Q.; Elstner, M.; Work, D. M.; J. Phys. Chem. 2005, 7, 3070.

24. Richard, R. M.; Ball, D.W.; J. Molec. Model, 2008, 14, 21.

25. Jalbout, A.F.; Darwish, A. M.; Alkahby, H. Y.; J. Mol. Struct. 2002, 585 , 205.

26. Wilcox, C. F.; Attygalle, A. B.; J. Mol. Struct. 1998, 434, 207.

27. Zheng-Xin, T.; Xiao-Hong, L.; Xian-Zhou, Z.; J. Mol. Struct. 2009, 907, 126.

28. Jursic, B. S.; J. Mol. Struct. 1998, 422, 253.

29. Zhao, J.; Cheng, X.; Yang, X.; J. Mol. Struct. 2006, 766, 87.

30. Jalbout, A. F.; Fernandez, S.; Chen, H.; J. Mol Struct. 2002, 584, 143.

31. Miller, T. M.; Arnold, S. T.; Viggiano, A. A.; Int. J. Mass Spectrom. 2003, 227, 413.

32. Namazian, M.; Siahrostami, S.; Coote, M. L.; J. Fluorine Chem. 2008, $129,222$.

33. Wang, L.; Int. J. Mass Spectrom. 2007, 264, 84.

34. Curtiss, L. A.; McGrath, M. P.; Blaudeau, J-P.; Davis, N. E.; Binning, R. C. Jr.; Radom, L.; J. Chem Phys. 1995, 103, 6104.

35. Xie, J.; Zare, R. N.; Chem. Phys. Lett. 1989, 159, 399.

36. Warshel, A.; Karplus, M.; J. Am. Chem. Soc. 1972, 94, 5612.

37. Warshel, A.; Levitt, M.; J. Mol. Biol. 1976, 103, 227.

38. Field, M. J.; Bash, P. A.; Karplus, M.; J. Comput. Chem. 1990, 11, 1970.

39. Maseras, F.; Morokuma, K.; J. Comput. Chem. 1995, 16, 1170.

40. Svensson, M.; Humbel, S.; Froese, R. D. J.; Matsubara, T.; Sieber, S.; Morokuma, K.; J. Phys. Chem. 1996, 100, 19357.

41. Dapprich, S.; Komaromi, I.; Byun, K. S.; Morokuma, K.; Frisch, M. J.; J. Mol. Struct. 1999, 1, 461.

42. Thomas, L. H.; Proc. Comb. Phil. Soc. 1927, 23, 542; Fermi, E.; Rend. Accad. Lincei 1927, 6, 602.

43. Hohenberg, P.; Kohn,W.; Phys. Rev. B 1964, 136, 864.

44. Kohn, W.; Sham, L. J.; Phys. Rev. 1965, 140, 1133.

45. Dykstra, C. E.; Frenking, G.; Kim, K. S.; Scuseria, G. E.; Theory and Applications of Computational Chemistry: The First Forty Years, Elsevier: Amsterdan, 2005.

46. Zhang, I. Y.; Luo, Y.; Xu, X.; J Chem Phys. 2010, 133, 104105.

47. Bensasson, R. V.; Zoete, V.; Dinkova-Kostova, A. T.; Talalay, P.; Chem. Res. Toxicol. 2008, 21, 805.

48. Librando, V.; Alparone, A.; Tomaselli, G.; J. Molec. Model. 2008, 12, 489.

49. Higashi, N.; Tanimoto, K.; Nishioka, M.; Ishikawa, K.; Taya, M.; J. Biochem. 2008, 144, 77 .

50. Morgan, J. E.; Gennis, R. B.; Maeda, A.; Photochem. Photobiol., B 2008, 84, 1038.

51. Bhattacharya, S. K.; Kshirsagar, A.; Eur. Phys. J. D. 2008, 48, 355.

52. Reinard, M. S.; Johnston, M. V.; J. Am. Soc. Mass Spectrom. 2008, 19, 389.

53. Frisch, M. J.; Trucks, G. W.; Schlegel, H. B.; Scuseria, G. E.; Robb, M. A.; Cheeseman, J. R.; Montgomery, Jr., J. A.; Vreven, T.; Kudin, 
K. N.; Burant, J. C.; Millam, J. M.; Iyengar, S. S.; Tomasi, J.; Barone, V.; Mennucci, B.; Cossi, M.; Scalmani, G.; Rega, N.; Petersson, G. A.; Nakatsuji, H.; Hada, M.; Ehara, M.; Toyota, K.; Fukuda, R.; Hasegawa, J.; Ishida, M.; Nakajima, T.; Honda, Y.; Kitao, O.; Nakai, H.; Klene, M.; Li, X.; Knox, J. E.; Hratchian, H. P.; Cross, J. B.; Bakken, V.; Adamo, C.; Jaramillo, J.; Gomperts, R.; Stratmann, R. E.; Yazyev, O.; Austin, A. J.; Cammi, R.; Pomelli, C.; Ochterski, J. W.; Ayala, P. Y.; Morokuma, K.; Voth, G. A.; Salvador, P.; Dannenberg, J. J.; Zakrzewski, V. G.; Dapprich, S.; Daniels, A. D.; Strain, M. C.; Farkas, O.; Malick, D. K.; Rabuck, A. D.; Raghavachari, K.; Foresman, J. B.; Ortiz, J. V.; Cui, Q.; Baboul, A. G.; Clifford, S.; Cioslowski, J.; Stefanov, B. B.; Liu, G.; Liashenko, A.; Piskorz, P.; Komaromi, I.; Martin, R. L.; Fox, D. J.; Keith, T.; Al-Laham, M. A.; Peng, C. Y.; Nanayakkara, A.; Challacombe, M.; Gill, P. M. W.; Johnson, B.; Chen, W.; Wong, M. W.; Gonzalez, C.; Pople, J. A.; Gaussian 03, Revision E.02; Gaussian, Inc., Wallingford CT, 2004.
54. Straatsma, T. P.; Aprà, E.; Windus, T. L.; Bylaska, E. J.; de Jong, W.; Hirata, S.; Valiev, M.; Hackler, M.; Pollack, L.; Harrison, R.; Dupuis, M.; Smith, D. M. A.; Nieplocha, J.; Tipparaju V.; Krishnan, M.; Auer, A. A.; Brown, E.; Cisneros, G.; Fann, G.; Früchtl, H.; Garza, J.; Hirao, K.; Kendall, R.; Nichols, J.; Tsemekhman, K.; Wolinski, K.; Anchell, J.; Bernholdt, D.; Borowski, P.; Clark, T.; Clerc, D.; Dachsel, H.; Deegan, M.; Dyall, K.; Elwood, D.; Glendening, E.; Gutowski, M.; Hess, A.; Jaffe, J.; Johnson, B.; Ju, J.; Kobayashi, R.; Kutteh, R.; Lin, Z.; Littlefield, R.; Long, X.; Meng, B.; Nakajima, T.; Niu, S.; Rosing, M.; Sandrone, G.; Stave, M.; Taylor, H.; Thomas, G.; van Lenthe, J.; Wong, A.; Zhang, Z.; NWChem, A Computational Chemistry Package for Parallel Computers, Version 4.6 (2004), Pacific Northwest National Laboratory, Richland, Washington 99352-0999, USA; Kendall, R. A.; Aprà, E.; Bernholdt, D. E.; Bylaska, E. J.; Dupuis, M.; Fann, G. I.; Harrison, R. J.; Ju, J.; Nichols, J. A.; Nieplocha, J.; Straatsma, T. P.; Windus, T. L.; Wong, A. T.; Computer Phys. Commun. 2000, 128, 260. 